\title{
Characterization of Individual Nanoparticles with Nanoprojectile-SIMS
}

\author{
A. B. Clubb, C. K. Liang, S. V. Verkhoturov, E. A. Schweikert \\ Department of Chemistry, Texas A\&M University, College Station, Texas, United States
}

The uniqueness of nanoparticles, NPs, due to their functionalities not present in bulk size is well documented. A nuanced understanding of their functionalities depends on their accurate characterization. Generally the analysis is done on an ensemble of NPs. However, there is likely some variation in the NP population and ensemble averaging can limit insight into the relationships between size, composition, and chemical reactivity of NPs. This is especially the case for very small NPs where minor changes in size, shape, or composition can significantly affect chemical reactivity. We have developed an innovative methodology for analyzing individual NPs, in particular objects of 2-50 nm in size.

Our approach is based on an innovative Secondary Ion Mass Spectrometry, SIMS, technique where NPs dispersed on a substrate are probed one by one. They are bombarded with a sequence of individual "nanoprojectiles", specifically $\mathrm{Au}_{400}^{4+}$ (a NP of $\sim 2 \mathrm{~nm}$ in diameter). The impact of a single nanoprojectile at hypervelocity $\sim 30 \mathrm{~km} / \mathrm{s}$ ) generates abundant ion emission from an area of $\sim 10 \mathrm{~nm}$ in diameter. The ejecta from each impact are mass-analyzed and recorded individually. This approach avoids problems due to ensemble averaging. From the pool of individual NP records (typically $\sim 1 \mathrm{M}$ ) we extract subsets of data from alike NPs; specifically we can identify molecules co-located within $\sim 10 \mathrm{~nm}$.

The instrument used for the investigation of discrete nano-objects is a custom-built secondary ion mass spectrometer comprised of a Au-liquid metal ion source (Au-LMIS) used to generate a variety of gold clusters as projectiles. A specific cluster can be mass-selected by a Wien filter installed on a $120 \mathrm{kV}$ platform. Clusters are steered by a series of deflectors to the suspended target, biased at $\pm 10 \mathrm{kV}$, giving a total kinetic energy of 110-130 qkeV. Collimators and pulser plates in the pathway of the primary ions reduces the dose to a sequence of individual projectiles of $\sim 1000$ projectiles per second to meet the requirements of an event-by-event bombardment/detection mode. Sputtered ions and electrons are extracted from the impact area by $90 \%$ transmission grids maintained at ground. Electrons or protons are turned using a magnetic field to a microchannel plate (MCP) detector that triggers the timing of the time of flight measurement. Secondary ions proceed to an 8 anode detector which stops the timing measurement.

An isolated nano-object constitutes a finite system where the energy imparted by the projectile cannot be dissipated as in a semi-infinite solid. As a consequence sputter and ion yields are size-dependent ${ }^{1}$. The same holds for electron emission ${ }^{2}$. The case of the secondary ions is illustrated with ejecta from gold NPs where the yields are increased twofold for 15 and $20 \mathrm{~nm}$ NPs over that from bulk gold. Gold NPs with a diameter of $50 \mathrm{~nm}$ produce secondary ion yields similar to that of the bulk case, while smaller gold NPs $(2-10 \mathrm{~nm})$ show a reduction from the bulk emission yields ${ }^{3}$. Another study showed that the nature of the ejecta may also differ from bulk-like secondary ion emission ${ }^{4}$. 
The impact parameter is critical in the projectile-NP collision. On "large" NPs ( $\geq 20 \mathrm{~nm})$ impacts can be direct, grazing, or interfacial. In a direct impact, the $\mathrm{Au}_{400}^{4+}$ projectile hits the NP squarely and causes the emission of secondary ions from up to $10 \mathrm{~nm}$ in depth. A grazing impact probes a thinner section of the NP, and so can give information characteristic of the outer shell. An interfacial impact near both the NP of study and the substrate gives rise to information from both. Since is it possible to separate the different types of impact parameters based on their secondary ion emission, selections can be done that make it possible to distinguish outer shell and core mass peaks.

For samples with dimensions less than $20 \mathrm{~nm}$, impact parameters blur and substrate contributions become prominent. Concurrently the projectile range exceeds the NP diameter. Thus it becomes feasible to assay "small" NPs simultaneously via the (customary) reflection and transmission emissions. A dual ToF MS has been assembled, still using $\mathrm{Au}_{400}^{4+}$ as a projectile where after bombardment secondary ions are extracted in the transmission and reflection directions. The emitted electrons or protons are again turned to separate start detectors and the rest of the secondary ions impact a second stop detector. In this approach the NPs are dispersed on suspended graphene. By having start detectors on both the transmission and reflection side, it is possible to only count impacts that produce emission on both sides of the graphene support by running in a coincidence start mode, where signal must be observed on both start detectors simultaneously for the time of flight measurement to be triggered.

Due to the atomic thickness of graphene, substrate contributions to the overall mass spectra are greatly reduced compared to the use of bulk supports. Also, through the use of the dual mass spectrometers more information is collected per impact compared to the single ToF commonly used in SIMS. This is of importance as samples less than $20 \mathrm{~nm}$ are probed are completely fragmented when impacted. NPs as small as $2 \mathrm{~nm}$ in diameter, dispersed on graphene have been identified with the dual mass spectrometer. Initial results suggest that transmission/reflection emissions may be optimized by adjusting the substrate characteristics to the size and composition of the NP.

\section{References:}

[1] L. Yang, M. Seah, E. Anstis, I. Gilmore, and J. Lee. J. Phys. Chem. C. 116, (2012), p. 9311-9318.

[2] M. Eller, S. Verkhoturov, S. Della-Negra, and E. A. Schweikert. J. Phys. Chem. C. 114, (2010), p. 17191-17196.

[3] C.-K. Liang, S. Verkhoturov, L.-J. Chen, and E. A. Schweikert. Int. J. Mass spectrom. 334, (2013), p. $43-48$.

[4] V. Pinnick, S. Rajagopalachary, S.Verkhoturov, L. Kaledin and E. A. Schweikert. Anal. Chem. 80, (2008), p. 9052-9057.

Work supported by the National Science Foundation, Grant CHE-1308312. 